\title{
INCIDÊNCIA, FATORES DE RISCO E MORTALIDADE DE PACIENTES COM INJÚRIA RENAL AGUDA EM UNIDADE DE TERAPIA INTENSIVA
}

\author{
Wellington Pereira Lopes ${ }^{1}$, Karolyne Cortes Dresch ${ }^{2}$, Ana Maria Silva Camargo ${ }^{2,}$ Renata Videira \\ Andrade dos Santos ${ }^{2}$ \\ ${ }^{1}$ Universidade Estadual de Londrina - UEL, Programa de Pós-graduando em Residência Multiprofissional em Saúde da \\ Família, Londrina, PR. ${ }^{2}$ Universidade do Oeste Paulista - UNOESTE, Curso de Enfermagem, Presidente Prudente, SP.
}

\section{RESUMO}

Injuria Renal Aguda é caracterizada pela deficiência súbita na filtração e nas funções dos rins, a incidência da Injuria Renal Aguda varia de acordo com as condições clínicas dos pacientes, sendo maior em Unidades de Terapia Intensiva, sendo assim, a mortalidade desses pacientes varia entre 70 a $80 \%$. O objetivo do estudo foi identificar incidência, fatores de risco e mortalidade desses pacientes. Trata-se de uma pesquisa retrospectiva exploratória quantitativa. Os dados coletados foram analisados com procedimentos estatísticos e apresentados através de tabelas e gráficos. A metodologia utilizada foi: análise retrospectiva de prontuários de pacientes que evoluíram com a doença. Dos 359 prontuários, os resultados foram: 25 pacientes desenvolveram a doença totalizando uma incidência de $6,96 \%$, sendo $44 \%$ do sexo feminino e $56 \%$ do sexo masculino, com mortalidade de $72 \%$. Conclui-se que o número de óbitos poderia ser diminuído por meio de prevenção e manutenção da IRA e que a sua incidência está totalmente ligada ao grande numero de óbitos nas Unidades de Terapia Intensiva.

Palavras-chave: Insuficiência Renal Aguda; Unidade de Terapia Intensiva; Fatores de risco; Incidência; Mortalidade.

\section{INCIDENCE, RISK FACTORS AND MORTALITY OF PATIENTS WITH ACUTE RENAL INJURY IN INTENSIVE CARE}

\begin{abstract}
Acute Renal Injury is characterized by sudden deficiency in filtration and in the functions of mouthwashes, with an incidence of Acute Renal Injury, varying according to the clinical conditions of the patients, being greater in Intensive Care Units, so, one day? an $80 \%$. The study is separating analysis, risk factors and puppy these patients. This is a retrospective quantitative exploratory research. Data were consisted with statistical procedures and preferred through charts and graphs. The methodology used was: retrospective analysis of patients with evolutionary evolution with disease. Of the 359 results, the results were: 25 patients developed a disease totaling an average of $6.96 \%$, being $44 \%$ female and $56 \%$ male, with mortality of $72 \%$. It was concluded that the number of deaths was reduced for the means of prevention and maintenance of ARF and that its incidence is totally related to the large number of deaths in the Intensive Care Units Keywords: Acute Renal Failure; Intensive care unit; Risk factors; Incidence; Mortality.
\end{abstract}




\section{INTRODUÇÃO}

Injuria Renal Aguda (IRA) é caracterizada pela deficiência súbita na taxa de filtração glomerular e nas outras funções dos rins, acarretando desequilíbrio hidroeletrolítico, ácido-básico e na homeostase do organismo ${ }^{1-2}$.

As alterações podem ocorrer por: redução da perfusão renal e alterações na hemodinâmica renal, toxicidade tubular direta, toxicidade tubular por rabdomiólise, obstrução intratubular precipitada, nefrite intersticial alérgica e síndrome hemolítico-urêmico ${ }^{1}$.

A incidência da IRA varia de acordo com as condições clínicas dos pacientes, sendo maior em Unidades de Terapia Intensiva, devido o quadro clínico debilitado do mesmo, sendo assim, a mortalidade desses pacientes varia entre 70 a $80 \%$, em um estudo houve o resultado de $76 \%$ e que os níveis elevados de creatinina sérica estão diretamente ligados à mortalidade dos pacientes acometidos pela doença ${ }^{3-4}$.

Os pacientes internados em uma Unidade de Terapia Intensiva são mais susceptíveis devido as complicações patológicas que propiciam a evolução da Injúria Renal Aguda, como por exemplo, insuficiência cardíaca, hipovolemia, pós-trauma, pós-cirúrgico, idade avançada, diabetes mellitus, trombose na veia renal, presença de oligoâmnnio, doenças do trato urinário além do uso crônico de anti-inflamatórios não hormonais entre outras ${ }^{1-5}$.

A etiologia da IRA consiste em pré-renal, intra-renal e pós-renal, respectivamente, a primeira se trata de uma resposta fisiológica ocasionada por uma diminuição da volemia ou uma isquemia renal a segundo por problemas intrínsecos e a última, lesão pós renal, é caracterizada por obstrução dos canais coletores até o orifício uretral externo devido a cálculos, coágulos, hipertrofia prostática entre outras ${ }^{9}$.

A mortalidade continua elevada e mesmo atualmente tendo um suporte maior de tratamento aos pacientes, podendo fazer com que ele consiga sobreviver mais tempo, isso não fez com que reduzisse as taxas de mortalidade causadas pela IRA ${ }^{6-7}$.

Este estudo teve como objetivo identificar incidência, fatores de risco e mortalidade de pacientes com Injúria Renal Aguda em Unidade de Terapia Intensiva.

\section{METODOLOGIA}

Estudo retrospectivo exploratório quantitativo, que foi realizado em uma Unidade de Terapia Intensiva de um hospital de grande porte situado no Oeste Paulista. Este estudo foi aprovado pelo Comitê de Ética em Pesquisa (CEP) da Universidade do Oeste Paulista (CAAE no 50403615.5.0000.5515).

Foram analisados 359 prontuários dos pacientes admitidos no local, no período de janeiro a junho de 2015, com base nos critérios de inclusão e exclusão previamente delimitados. Foram utilizados como critérios de inclusão: pacientes que adquiriram Injúria Renal Aguda na Unidade de Terapia Intensiva. E como critérios de exclusão: pacientes que já eram portadores da Insuficiência Renal Crônica antes de adentrarem na Unidade de Terapia Intensiva e em tratamento dialítico crônico, menores de 18 anos e que adquiriram a IRA fora da data do levantamento de dados.

Utilizou-se instrumento próprio para coleta de dados, sendo este constituído de 30 itens, prontuário, nome, sexo, idade, procedência, diagnóstico de entrada, data de entrada, data de diagnóstico da IRA, níveis de creatinina, ureia e potássio na entrada e no diagnóstico, fatores de risco como uso de ventilação mecânica, drogas vasoativas, choque séptico, diabetes, hipertensão arterial, insuficiência cardíaca crônica, uso de anti-inflamatório não esteroidal (AINE) no domicílio, oligúria, medidas de pressão venosa central (PVC), alta e óbito.

Os dados foram submetidos à análise estatística descritiva e apresentados em tabelas na forma de frequência absoluta e percentual. As análises estatísticas foram realizadas no programa SPSS 21.0, sendo considerada aceita a hipótese de que os óbitos dos pacientes no estudo são 
influenciados pelas outras variáveis em estudo, quando a probabilidade for menor ou igual a 0,05 $(p<0,05)$. Quando o teste do Qui-quadrado apresentou significância estatística utilizou-se o procedimento de Marascuilo como o teste de comparações múltiplas para estabelecer as categorias que diferem entre si.

\section{RESULTADOS}

Durante o período de coleta de dados foram analisados 359 prontuários dos pacientes admitidos no local, no período de janeiro a junho de 2015, dos quais 25 desenvolveram Injúria Renal Aguda, mostrando uma incidência total de 6,96\% nessa UTI.

Nos dados observados da amostra estudada, $56 \%$ são do sexo masculino e $44 \%$ do sexo feminino, embora os dados obtidos não tenham p-valor significativo, encontramos que a diferença de sexo pode predispor a ocorrência de IRA nos indivíduos internados.

Tabela 1. Cruzamento entre fatores de risco com o prognóstico, dos pacientes com Injúria Renal Aguda em Unidade de Terapia Intensiva.

\begin{tabular}{|c|c|c|c|}
\hline & Alta médica & Alta Óbito & p-valor \\
\hline Uso de Ventilação Mecânica & $71,4 \%$ & $88,9 \%$ & 0,285 \\
\hline Drogas vasoativas & $57,1 \%$ & $88,9 \%$ & 0,075 \\
\hline Choque séptico & $57,1 \%$ & $38,9 \%$ & 0,409 \\
\hline Diabetes & $57,1 \%$ & $38,9 \%$ & 0,409 \\
\hline Hipertensão arterial & $57,1 \%$ & $44,4 \%$ & 0,568 \\
\hline Insuficiência cardíaca crônica & $14,3 \%$ & $11,1 \%$ & 0,826 \\
\hline Uso de AINE no Domicílio & $0,0 \%$ & $5,6 \%$ & 0,524 \\
\hline Oligúria & $42,9 \%$ & $88,9 \%$ & $0,016^{*}$ \\
\hline
\end{tabular}

Com relação aos fatores de risco para Injúria Renal Aguda a Tabela 4 mostra que apenas o fator de risco oligúria apresentou dependência com o prognóstico, sendo assim, pelo Teste de Quiquadrado há evidências de que o prognóstico tem alguma relação de dependência com a presença da oligúria. 
Tabela 2. Prognósticos e quantidade de dias até o prognóstico. Presidente Prudente, 2015.

\begin{tabular}{|l|l|l|l|}
\hline Prognóstico & $(\mathrm{n}=25)$ & $\%$ & $\begin{array}{l}\text { Dias até prognóstico } \\
\text { Média ( } \pm \text { Desvio Padrão) }\end{array}$ \\
\hline Alta Médica & 7 & $28,0 \%$ & $27,71( \pm 24,83)$ \\
\hline Alta Óbito & 18 & $72,0 \%$ & $9,78( \pm 7,84)$ \\
\hline Total & 25 & $100,0 \%$ & $14,80( \pm 16,29)$ \\
\hline
\end{tabular}

O prognóstico da população estudada, média e o desvio padrão de dias até o prognóstico, em geral, os pacientes ficam internados 15 dias com um desvio de mais ou menos 16 dias, quanto aos pacientes que obtiveram alta médica, eles ficam internados em média 28 dias com um desvio de mais ou menos 25 dias. Para os pacientes que vieram a óbito, estes ficam internados em média 10 dias com um desvio de 8 dias para mais ou para menos.

\section{DISCUSSÕES}

Dos 359 prontuários analisados, 25 pacientes desenvolveram Injúria Renal Aguda totalizando uma incidência de $6,96 \%$, sendo $44 \%$ do sexo feminino e $56 \%$ do sexo masculino, corroborando com outros estudos utilizados nas discussões. Quanto aos fatores de risco o que mais teve relevância estatística pelo Teste de Qui-quadrado foi a oligúria com p-valor 0,016 . Foi observado que o uso de drogas vasoativas e ventilação mecânica permaneceram elevados nos resultados da amostra, tanto naqueles que receberam alta médica como nos que evoluíram a óbito, porém não obtiveram p-valor significativo. A mortalidade encontrada foi de $72 \%$, estando de acordo com as literaturas utilizadas na elaboração como nas discussões.

A incidência varia de acordo com as condições clínicas dos pacientes, a incidência de pacientes críticos internados em UTI, que evoluem para IRA varia entre $17 \%-35 \%{ }^{7}$, e nos demais setores hospitalares essa incidência ocorre em cerca de $1,9 \%{ }^{10}$. Se comparado ao presente estudo, esses valores encontram-se acima dos resultados obtidos nesta pesquisa.

Indo de encontro com o estudo, acredita-se que a incidência de IRA em ambientes críticos como as UTI's ocorre em aproximadamente 1 - $5 \%$ dos indivíduos ali internados ${ }^{9}$.

Mulheres geralmente possuem mais proteção em comparação aos homens pela inibição de estrogênios e ativação de androgênios, sendo assim, apresentam menor risco para doença renal ${ }^{11-}$ 12

Existe uma nítida diferença na prevalência de Hipertensão Arterial Sistólica (HAS) relacionada à idade e gênero. A pressão arterial diastólica tende a ser sempre menor nas mulheres, independentemente da faixa etária ${ }^{13}$.

Com a pressão elevada, em torno de $150 \mathrm{mmHg}$, o débito renal de água e sal aumenta três vezes mais que a ingestão, sendo assim o corpo perde líquido, e o volume sanguíneo e pressão arterial diminuem. Esse mecanismo não cessa enquanto a pressão arterial não atingir o ponto de equilíbrio ${ }^{14}$.

Em relação ao resultado obtido referente à idade dos portadores de IRA, que 52\% têm idade menor que 60 anos, não corrobora com os dados dos demais estudos utilizados para as discussões, onde estatisticamente os resultados revelam que pessoas com idade avançada, acima de 60 anos, tendem a desenvolver IRA devido as co-morbidades que ocorrem nessa faixa etária, e possuem relação direta com o funcionamento renal ${ }^{15}$. 
Como apresentado na tabela 1 existem fatores que em muitos estudos são apontados como concomitantes a IRA e que de alguma forma aumentam consistentemente a mortalidade causada por esta patologia. Fatores como o uso de ventilação mecânica e drogas vasoativas, doenças cardiovasculares e sepse estão associados a gravidade da IRA, que eleva a mortalidade dos pacientes apesar dos ajustes para a comorbidade ${ }^{15-16}$.

O uso de drogas vasoativas em pacientes com IRA, após ressuscitação volêmica é controverso e polêmico, porem estudos apontam que tanto do ponto de vista geral e inclusive renal a restauração da pressão arterial dentro dos valores de auto reguladores deverá ocorrer prontamente com tais drogas trazendo um efeito benéfico ${ }^{10}$.

A IRA oligúrica é considerada quando ocorre uma redução no fluxo de diurese, sendo considerados valores entre 100 a $400 \mathrm{ml} / 24 \mathrm{~h}$. O baixo fluxo urinário indica que o escasso filtrado glomerular é de alguma forma reclamado em sua quase totalidade. Essa disfunção tubular deve-se ao fato de em vários pontos do rim as células tubulares apresentarem-se necrosadas ou ausentes, restando aos túbulos, em certos segmentos, apenas a membrana basal ${ }^{9-11}$.

Enquanto na forma oligúrica o fluxo urinário é usualmente inferior a $100 \mathrm{ml} / \mathrm{dia}$, é comum na forma não oligúrica a presença de débitos urinários elevados, às vezes superiores a $2 \mathrm{~L} / \mathrm{dia}$, indicando que, nessa forma de IRA, a recuperação da pequena quantidade de filtrado que se forma é apenas parcial. A distinção entre as duas formas de IRA é importante por duas razões: em primeiro lugar, o prognóstico da IRA não oligúrica é melhor do que o da forma oligúrica. Em segundo lugar, o controle do balanço hídrico é mais fácil na forma não oligúrica do que na oligúrica, uma vez que é menor a tendência à retenção de volume ${ }^{8}$ A IRA não oligúrica é vista, frequentemente, em pós-cirúrgicos, traumas, hipotensão, nefrotoxinas e rabdomiólise. Sua incidência gira em torno de 25 a $80 \%$ de todos os casos de IRA e pode passar despercebida, se somente for avaliada a diurese ${ }^{12}$.

Conforme dados da tabela 2, observa-se que $28 \%$ dos pacientes que obtiveram Injúria Renal Aguda conseguiram se recuperar desse quadro e receberam alta da Unidade de Terapia Intensiva, entretanto, de acordo com Ponce $50 \%$ dos pacientes que adquirem IRA tem um bom prognóstico e conseguem se recuperar dessa patologia ${ }^{13}$.

\section{CONCLUSÃO}

Com base nos dados apresentados, conclui - se que a prevenção e a manutenção da IRA é de extrema importância na Unidade de Terapia Intensiva, e que sua incidência está diretamente ligado a grande chance de óbito e complicações do paciente, sendo assim, como forma de redução de danos e prevenção de novos casos de IRA no setor, medidas podem ser tomadas sendo elas: a preparação e informação dessa equipe sobre a importância do monitoramento do balanço hídrico, estarem atentos a sinais de desidratação e alterações hemodinâmicas, realizarem verificação da PVC ou DELTA PP (delta = variação; PP = pressão de pulso), conforme a disponibilidade dos equipamentos no local e discussões com a equipe multiprofissional. Manter a pressão arterial média em $80 \mathrm{~mm} / \mathrm{hg}$, observar de forma constante o débito urinário, fornecer hidratação adequada, atenção especial aos pacientes com pancreatite pós cirúrgico e politraumatizados, cuidado na administração de anti-inflamatórios e avaliar os protocolos implantados nos serviços de saúde com estudos e pesquisas buscando reduzir o agravamento dos casos e reduzindo o número de óbitos. 


\section{DECLARAÇÃO DE CONFLITO DE INTERESSE}

Os autores declaram não haver qualquer potencial conflite de interesse que possa interferir na imparcialidade deste trabalho científico.

\section{REFERÊNCIAS}

1.Riella, MC. Princípios de nefrologia e distúrbios hidroeletrolíticos. 5. ed. Rio de Janeiro: Guanabara Koogan, 2014. 1033.

2. Bernardina, LD. et al. Evolução clínica de pacientes com insuficiência renal aguda em unidade de terapia intensiva. Acta Paulista de Enfermagem, São Paulo, v. 21, n. spe, p.174-178, 2008.

3. Nunes, TF. et al. Insuficiência renal aguda. Medicina, Ribeirão Preto, v. 43, n. 3, p. 272-282, 2010.

4. Ponce, D. et al. Injúria renal aguda em unidade de terapia intensiva: estudo prospectivo sobre a incidência, fatores de risco e mortalidade. Rev. Bras. Ter. Intensiva, São Paulo, v. 23, n. 3, p. 321326, set. 2011.

5. Costa, JAC.; NETO OM.; NETO MM. Insuficiência renal aguda. Medicina, Ribeirão Preto, v. 36, p. 307-324. abr./dez. 2003.

6. Magro, MCS.; VATTIMO MFF. Avaliação da função renal: creatinina e outros biomarcadores*. Rev. Bras. Ter. Intensiva, São Paulo, v. 19, n. 2, p. 182-185, mai. 2007.

7. Luft, J. et al. Lesão renal aguda em unidade de tratamento intensivo: característica clínicas e desfechos. Cogitare Enfermagem, v. 21, n. 2, 2016.

9. Padilha, KG. et al. Enfermagem em UTI: cuidando do paciente crítico. 1. ed. Barueri: Manole, p 1446. 2010.

10. Santos, ER. Associação do RIFLE com letalidade e tempo de internação em pacientes críticos com lesão renal aguda. Rev. Bras. Ter. Intensiva, São Paulo, v. 21, n. 4, p. 359-368, dec. 2009.

11. Kang, AK.; MILLER, JA. Impact of gender on renal disease: the role of the renin angiotensin system. ClinInvest Med., v. 26, n.1, p.38-44, 2003.

12. Silva, AVE. Implementação da classificação Kdigo para avaliação da função renal no pósoperatório de cirurgia geral. São Paulo: UNB, 2014.

13. Colombo, FC. Hipertensão arterial na mulher. In: PAOLA AAV.; BARBOSA MM.; GUIMARÃES JI. Cardiologia - Livro Texto da Sociedade Brasileira de Cardiologia. São Paulo: Manole, 2011. p. 62830.

14. Hall JE. Guyton \& Hall: Tratado de fisiologia médica. 12. ed. Rio de Janeiro: Elsevier, 2011.

15. Vieira JR, JM. et al. Effect of acute kidney injury on weaning from mechanical ventilation in critically ill patients. Critical Care Medicine, v. 35, n. 1, p. 184-191, 2007. https://doi.org/10.1097/01.CCM.0000249828.81705.65

16. Bellomo, R.; WAN, L.; MAY, C. Vasoactive drugs and acute kidney injury. Critical Care Medicine, 
v. 36, n. 4, p. S179-S186, 2008. https://doi.org/10.1097/CCM.0b013e318169167f 